\title{
Clinical Characteristics of 8 Sporadic Cases of Community- Acquired Legionella Pneumonia in Advanced Age
}

\author{
Eiichiro Sugihara ${ }^{1}$, Takashi Dambara ${ }^{1}$, Miyoji Aiba ${ }^{2}$, Masaki Okamoto ${ }^{3}$, Junko Yonemitsu ${ }^{3}$, \\ Satoshi Sonobe ${ }^{3}$, Hideyuki Koga ${ }^{3}$, Akihiro Inui ${ }^{1}$, Naoko Hada ${ }^{1}$, Naomi Matsumoto ${ }^{1}$, \\ Yoko Goto ${ }^{1}$, Toshio Naito ${ }^{1}$, Hiroshi Isonuma ${ }^{1}$ and Yasuo Hayashida ${ }^{1}$
}

\begin{abstract}
Legionella spp are well recognized as one of the etiologic factor in pneumonia, but it is difficult to distinguish the clinical features of Legionella infection from pneumonia due to other causes. The objective of the present study was to examine the clinical characteristics of community-acquired Legionella pneumonia in elderly patients. We reviewed the clinical, laboratory and radiographic findings in 8 patients diagnosed as having pneumonia caused by Legionella. The diagnosis was confirmed by the presence of urinary antigen, bacterial culture, polymerase chain reaction (PCR) and serum antibody. There were 6 men and 2 women, whose ages ranged from 76 to 85 years. All patients had fever and hypoxia, four patients had respiratory symptoms. The initial chest X-ray findings were varied-consolidation, ground glass opacity, pleural effusion and linear shadow. Urinary antigen was positive in 4 patients, bacterial culture in 2, PCR on the sputum in 3 and serum antibody in 2 patients. As pneumonia caused by Legionella often becomes life-threatening, especially in elderly people, it is imperative to diagnose it at the initial stage. In this study, urinary antigen proved to be the most useful diagnostic means. However, it is important to confirm the diagnosis through plural examinations.
\end{abstract}

Key words: Legionella pneumonia, community-acquired pneumonia, sporadic cases, elderly person

(DOI: 10.2169/internalmedicine.46.6084)

\section{Introduction}

The potential importance of Legionella species as the etiology of community-acquired pneumonia has been reported in the West $(1,2)$. Ishida et al (3) reported that in Japan, Legionella species was the causative pathogen in only about $0.6 \%$ of the cases of community-acquired pneumonia unlike the higher frequency reported in the West.

Whether it is possible to identify pneumonia caused by Legionella from the clinical features remains a matter of debate. Although there is no single clinical manifestation that distinguishes from other types of community-acquired pneumonia, it has been suggested that if there is a characteristic clinical profile, the likelihood of the diagnosis would increase (4-6). Thus, an accurate presumptive clinical diagnosis could be made on the basis of the proper association of key signs and symptoms. It is difficult to distinguish the clinical features of Legionella infection from other types of pneumonia, especially in sporadic cases. As the clinical condition of patient with $L$. pneumonia rapidly worsens, it is imperative to diagnose it at the initial stage and to control the infection with appropriate antibiotics.

We report the characteristics of 8 patients of advanced age with $L$. pneumonia. The purpose of this study was to contribute to a better understanding of $L$. pneumophilia pneumonia by examining the clinical features of thoracic infections caused by these pathogens in the elderly.

\section{Patients and Methods}

We retrospectively studied the clinical records of 8 patients of advanced age who were diagnosed with $L$. pneumonia. The diagnosis was based on the criteria proposed by

\footnotetext{
${ }^{1}$ Department of General Medicine, Juntendo University School of Medicine, Tokyo, ${ }^{2}$ Department of General Medicine, Juntendo Tokyo Koto Geriatric Medical Center, Tokyo and ${ }^{3}$ National Kyushu Respiratory Clinical and Reseach Center

Received for publication June 21, 2006; Accepted for publication December 22, 2006

Correspondence to Eiichiro Sugihara, es66@med.juntendo.ac.jp
} 
Table 1. Profile of the Patients with Legionella pneumonia

\begin{tabular}{|cccccc|}
\hline Case & Age & Sex & Underlying disease & Symptom§ & $\begin{array}{c}\text { Surmised source of } \\
\text { infection }\end{array}$ \\
\hline 1 & 84 & $M$ & $(-)$ & fever & unknown \\
\hline 2 & 81 & M & old tbc & fever, fatigue & unknown \\
\hline 3 & 79 & F & HT & fever, dyspnea & unknown \\
\hline 4 & 86 & F & HT & fever & unknown \\
\hline 5 & 81 & M & Depression & fever, dyspnea, sore throat & unknown \\
\hline 6 & 85 & M & HT, Renal failure & fever, cough, sputum, dyspnea unknown \\
\hline 7 & 81 & M & Emphysema & fever, cough, sputum, dyspnea unknown \\
\hline 8 & 76 & M & HT, DM & fever, consciousness loss & hot spring \\
\hline
\end{tabular}

Yamaguchi et al (7) in Japan. That is, 1) isolation of $L$. species from sputum or lung tissue, 2) positive urinary antigen, 3) high titer of serum antibody, 4) positive results of PCR. One positive finding among these four criteria, is sufficient to establish the diagnosis of $L$. pneumonia.

At the initial visit and after a complete clinical history taking, the patients were subjected to physical examination and biochemistry and hematologic tests. We also carried out bacteriological, urinary antigen, serological investigations and PCR on the sputum, for each patient.

\section{Bacteriological investigations}

Respiratory specimens containing mucus were liquefied with semi-alkaline protease, incubated at $37^{\circ} \mathrm{C}$ for $10 \mathrm{~min}$ utes and then centrifuged. Each specimen was incubated in BCYE $\alpha$ and WYO $\alpha$. The plates were incubated and were examined for 3 to 10 days. Visible colonies subcultured on BCYE $\alpha$ agar plates and $\mathrm{WYO} \alpha$ agar plates were considered as possible Legionella species and were tested by direct fluorescence-antibody assay (DFA) for L. pneumophila serogroups 1 to 6.

Polymerase chain reaction $(P C R)$

PCR was performed with primers previously determined to amplify a 386-bp product within the 16s rRNA gene of L. pneumophila in respiratory samples (8). It was reported that the sensitivity and specificity of respiratory section were 83 100\% and 90 100\%, respectively.

Urinary antigen and serological investigations

Legionella antigens in urine samples were examined by immunochromatographic assay (Binax, Portland, OR, USA) and urinary antigen enzyme immunoassay (Binax). The serum antibody titer was determined by the microplate agglutination test (Denka Seiken, Tokyo, Japan).

\section{Results}

Table 1 shows the profile of the present 8 patients. $L$. pneumophilia pneumonia infection was identified in 6 men and 2 women, whose ages ranged from 76 to 85 years. Seven patients had an underlying disease. All patients had fever, however, 4 patients had no respiratory symptoms such as cough, sputum or dyspnea. In one patient, we could surmise the source of infection (hot spring).

Table 2 shows the laboratory data on admission. All patients had hypoxia and a high CRP value. Six patients had a high WBC count. Three patients had a high CPK score, but no complicating rhabdomyolysis or acute renal failure. There were no patients with hyponatremia .

Treatment and outcome are shown in Table 3. Seven patients had been under treatment with antibiotics such as imipenem/cilastatin (IPM/CS), meropenem (MEPM), ceftazidime (CAZ), clindamycin (CLDM), cefozopran (CZOP), sultamicillin tosilate (SBTPC). As for the therapy for $L$. pneumophilia pneumonia, all patients were treated with two or more antibiotics, mainly ciprofloxacin (CPFX), erythromycin (EM) and refanpicin (RFP). A corticosteroid was used in 4 patients. With regard to their outcome, 6 patients were cured and two patients died.

Chest radiographic findings are shown in Table 4. Consolidation, which tended to be seen in peripheral lung, was present in 7 patients. Ground glass opacity was seen in 5 (in four it was located around the consolidation, and in one it was solitary). An interstitial shadow was seen in one patient. Pleural effusion was identified in 2 patients. No cavity or nodular infiltration was detected. Most patients showed a lobar pneumonia pattern. There were four patients who presented bilateral infiltrates and the radiographic shadow occupied more than one-third of the lung field.

The results of tests indicating a definite diagnosis are shown in Table 5. Gimenz staining was positive in 2 of 8 cases, sputum culture in 2 (L. pneumophila, serogroup 1) cases, urinary antigen in 4 cases, PCR on the sputum in 3, and serum antibody in 2 of 8 cases (L. pneumophila, serogroup 1, 256 titer).

\section{Discussion}

L. pneumophia pneumonia was first reported in Japan in 1981 by Saito et al. (9) Since then, several types of L. pneumophila pneumonia have been reported in Japan. Lieberman et al, (1) reported that $L$. species were the causative patho- 
Table 2. Laboratory Data on Admission

\begin{tabular}{|c|c|c|c|c|c|c|c|c|c|c|}
\hline Case & $\mathrm{WBC} / \mathrm{mm}^{3}$ & CRP mg/dl & ESR mm/hr & $\mathrm{PaO} 2$ torr & $\mathrm{PaCO}$ torr & GOT IU/I & GPT IU/l & LDH IU/l & CPK IU/l & Na mEq \\
\hline 1 & 12000 & 21 & 12 & 58.5 & 31 & 146 & 89 & 737 & 356 & 141 \\
\hline 2 & 5200 & 16.3 & 62 & 49.7 & 36.3 & 77 & 45 & 435 & 110 & 143 \\
\hline 3 & 16600 & 23.8 & 147 & 58.3 & 32.5 & 36 & 17 & 485 & 135 & 134 \\
\hline 4 & 12500 & 20.7 & & 52.5 & 35.7 & 54 & 44 & 316 & 248 & 140 \\
\hline 5 & 12700 & 7.9 & & 62.8 & 33 & 26 & 14 & 245 & 39 & 155 \\
\hline 6 & 9700 & 18.8 & & 60.8 & 31 & 40 & 19 & 237 & & \\
\hline 7 & 11400 & 14.5 & & 48.6 & 30.3 & 322 & 186 & 838 & 671 & 155 \\
\hline 8 & 7000 & 30 & 117 & 58.1 & 30.3 & 23 & 27 & 255 & 83 & 137 \\
\hline
\end{tabular}

Table 3. Treatment and Outcome

\begin{tabular}{ccccc}
\hline Case & Pretreatment & Treatment & Supportive therapy & Outcome \\
\hline 1 & MEPM & CPFX+EM & $\begin{array}{c}\text { Mechanical Ventilation, } \\
\text { methylpredonisolone }\end{array}$ & Cure \\
2 & IPM/CS & CPFX+CAM & $(-)$ & Cure \\
3 & CAZ+CLDM & CPFX+EM & methylpredonisolone & Died \\
4 & $(-)$ & EM+RFP & $(-)$ & Cure \\
5 & IPM/CS+CLDM & EM+RFP+MINO & methylpredonisolone & Cure \\
6 & CZOP & CPFX+CAM+RFP & $(-)$ & Cure \\
7 & SBTPC+CLDM & CPFX+EM & methylpredonisolon & Died \\
8 & MEPM & EM+RFP & $(-)$ & Cure \\
\hline
\end{tabular}

CPFX : ciprofloxacin, EM : erythromycin, CAM : clarithromycin, $\mathrm{MINO}$ : minomycin, RFP : refanpicin,MEPM : meropenem, IPM/CS : imipenem/cilastatin, CAZ : ceftazidime, CZOP : cefozopran, SBTPC : sultamicillin tosilate, CLDM : clindamycin.

gens in $16.2 \%$ of the cases of community-acquired pneumonia in the West. However, the percentage in Legionella species causative of community-acquired pneumonia is very low in Japan. It might be suggested that sporadic cases of $L$. pneumophila pneumonia must be overlooked without a definite diagnosis.

In general, L. pneumophila pneumonia presents with various symptoms: hyponatremia, impaired level of consciousness, cough, sputum, fever, diarrhea, or a combination of these symptoms (10). In the early phase of L. pneumophila pneumonia, $10 \%$ of the chest X-rays taken are almost normal (11). According to Heath et al (12), eight of their 39 patients did not have severe a pneumonia. Although L. pneumophila pneumonia tends to cause severe pneumonia, some patients improve if given appropriate therapy at an early stage. Thus, an early diagnosis is of great importance, we have described the characteristics of 8 elderly patients with L. pneumophila pneumonia.

In this study, all patients had hypoxia and fever. However, there were no specific laboratory data or clinical features in the initial stage. It was our impression that hypoxia was severe, given their shadow on chest X-ray and CT findings. From the viewpoint of differential diagnosis, bacterial pneumonia caused by Streptococcus pneumoniae or other bacterial infection might be more important than atypical pneumonia. In four patients, although they had severe pneumonia and hypoxia, no respiratory symptoms such as, cough, sputum, dyspnea, etc, were observed. Thus, when there are no respiratory symptoms other than fever, chest X-ray might not be taken.

It was reported that the delay in appropriate therapy, an underlying disease (cancer, diabetes, renal disease, etc.) and 
Table 4. Chest Radiographic Findings

\begin{tabular}{l|c}
\hline & No. of patients \\
\hline Location & 4 \\
$\quad$ bilateral infiltrate & 3 \\
$\quad$ right lung infiltrate & 1 \\
$\quad$ left lung infiltrate & \\
Extent of radiografhic shadow expressed as percent of one lung field & 2 \\
$<1 / 3$ & 2 \\
$1 / 3 \sim 2 / 3$ & 4 \\
$2 / 3<$ & 0 \\
Cavity & 2 \\
Pleural effusion & 6 \\
Lobar pneumonia & 1 \\
Interstitial pneumonia & \\
Characteristics of opacity & 2 \\
$\quad$ Air-space consolidation & 4 \\
$\quad$ Mixed air space consolidation and ground glass opacity & 1 \\
$\quad$ Ground glass opacity & \\
\hline
\end{tabular}

Table 5. The Methods for Definite Diagnosis

\begin{tabular}{cccccc}
\hline Case & Gimenez stain & Sputum culture & PCR on sputum & Urinary antigen & Serum antibody \\
\hline 1 & $(+)$ & $(+)$ & $(-)$ & $(-)$ & $(-)$ \\
& & L. pneumophila (serogroup 1) & & & \\
2 & $(-)$ & $(-)$ & $(+)$ & $(-)$ & $(+) 256$ \\
3 & $(-)$ & $(-)$ & $(-)$ & $(-)$ & $(+) 256$ \\
4 & $(-)$ & $(-)$ & $(+)$ & $(-)$ & $(-)$ \\
5 & $(-)$ & $(-)$ & $(-)$ & $(+)$ & $(-)$ \\
6 & $(+)$ & $(+)$ & $(+)$ & $(+)$ & $(-)$ \\
& & L.pneumophila (serogroup 1) & & $(-)$ & $(+)$ \\
7 & $(-)$ & $(-)$ & $(-)$ & $(+)$ & $(-)$ \\
8 & $(-)$ & $(-)$ & & & \\
\hline
\end{tabular}

age were important risk factor for L. pneumophila pneumonia $(10,12,13)$. In this study, the average number of days from onset to appropriate therapy was 5.0. Six of these 8 patients were cured regardless of their advanced age and existing underlying disease. The number of days to appropriate therapy of 2 patients who died cases (cases 3 and 7) was 8, which was a delayed timing compared to other cases. We found the prognosis was associated with the delay in appropriate therapy. There was not obvious relationship between the use of methylpredonisolone and outcome.

There have been numerous reports with regard to the imaging findings of $L$. pneumophila pneumonia. The pulmonary infiltrates seen in $L$. pneumophila pneumonia are predominantly seen in the lower lobe, sometimes bilaterally, and there is rapid progression and pleural effusion $(11,14$, $15)$. In the present study, we found a similar distribution of pulmonary infiltrates and frequency of pleural effusion com- pared to previous reports. Yagyu et al (10) reported the following imaging findings of $L$. pneumophila pneumonia: multiple segments were affected and peripheral lung consolidation with ground glass opacity was present. Ground glass opacity was mainly located around the consolidation. In our study, there were four patients who had a similar pattern.

In establishing the definite diagnosis of L. pneumophila pneumonia, either a bacteriological test, serological examination, urinary antigen or PCR for Legionella is needed. There have been reports describing the usefulness of urinary antigen for the diagnosis of Legionnaire's disease (16-18). Urinary antigen was positive in 4 of 8 cases (positive rate $50 \%$ ) in the present study. Urinary antigen might be the most useful for the initial diagnosis of L. pneumophila pneumonia. Although the positive rate of urinary antigen was higher compared with other examinations, there were patients who tested positive only for other examinations. We 
consider that each examination was valuable and that they should be carried out when encountering patients with severe pneumonia.

In elderly patients with $L$. pneumophila pneumonia may present as fever without respiratory symptoms. The chest Xray findings might show a bilateral distribution, multiple segments might be affected, and peripheral consolidation with ground glass opacity is observed. As L. pneumophila pneumonia often becomes a life-threatening condition, it is critical to diagnose it in its initial stage. The key principles of management of L. pneumophila pneumonia are early diagnosis and control of the infection with appropriate antibiotics. It is important to be aware of the fact that elderly patients with $L$. pneumophila pneumonia might not present respiratory symptoms and that for an early diagnosis plural examinations will be needed. A detailed analysis of the clinical features might contribute to the diagnosis of $L$. pneumophila pneumonia.

\section{References}

1. Lieberman D, Porath A, Schalaeffer F, Lieberman D, Boldur I. Legionella species community-acquired pneumonia. A review of 56 hospitalized adult patients. Chest 109: 1243-1249, 1996.

2. McNally C, Hacman B, Fields BS, Plouffe JE. Potential importance of Legionella species as etiologies in community-acquired pneumonia (CAP). Diagn Microbiol Infect Dis 38: 79-82, 2000.

3. Ishida $T$, Hashimoto $T$, Arita M, Ito I, Osawa M. Etiology of community-acquired pneumonia in hospitalized patients: A 3-year prospective study in Japan. Chest 114: 1588-1593, 1998.

4. Edelstein PH. Legionnaires' disease. Clin Infect Dis 16: 741-747, 1993.

5. Roig J, Domingo C, Morera J. Legionnaires' disease. Chest 105: 1817-1825, 1994.

6. Stout JE, Yu VL. Legionellosis. N Engl J Med 337: 682-687, 1997.

7. Yamaguchi K, Tateda K, Ishii Y, et al. Clinical and diagnostic characteristics of Legionella pneumonia. Kansenshogaku Zasshi 71: 634-643, 1997 (in Japanese).

8. Cloud JL, Carroll KC, Pixton P, Erali M, Hillyard DR. Detection of Legionella species in respiratory specimens using PCR with sequencing confirmation. J Clin Microbiol 38: 1709-1712, 2000.

9. Saito A, Shimoda T, Nagasawa M, et al. The first case of Legionnaires' disease in Japan. Kansenshogaku Zasshi 55: 124-128, 1981 (in Japanese).

10. Yagyu $H$, Nakamura $H$, Tuschida F, et al. Chest CT findings and clinical features in mild Legionella pneumonia. Intern Med 42:
477-482, 2003.

11. Tan MJ, Tan JS, Hamor RH, File TM Jr, Breiman RF. The radiographic manifestations of Legionnaire's disease. The Ohio Community-Based Pneumonia Incidence Study Group. Chest 117: 398-403, 2000.

12. Heath CH, Grove DI, Looke DFM, et al. Delay in appropiate therapy of Legionalla pneumonia associated with increased mortality. Eur. J Clin Microbiol Infect Dis 15: 286-290, 1996.

13. Marston BJ, Lipman HB, Breiman RF. Surveillance for Legionnaires' disease. Risk factors for morbidity and mortality. Arch Intern Med 154: 2417-2422, 1994.

14. Kroboth FJ, Yu VL, Reddy SC, Yu Ac. Clinicoradiographic correlation with the extent of Legionnaire disease. AJR Am J Roentgenol 141: 263-268, 1983.

15. Dietrich PA, Johnson RD, Fairbank JT, Walke JS. The chest radiograph in Legionnaires' disease. Radiology 127: 577-582, 1982.

16. Dominguez J, Gali N, Blanco S, et al. Assessment of a new test to detect Legionella urinary antigen for the diagnosis of Legionnaires' Disease. Diagn Microbiol Infect Dis 41: 199-203, 2001.

17. Sopena N, Sabria M, Pedro-Botet ML, et al. Factors related to persistence of Legionella urinary antigen excretion in patients with Legionnaires' disease. Eur J Clin Microbiol Infect Dis 21: 845848, 2002.

18. Waterer GW, Baselski VS, Wunderink RG. Legionella and community-acquired pneumonia: A review of current diagnostic tests from a clinician's viewpoint. Am J Med 110: 41-48, 2001.

(C) 2007 The Japanese Society of Internal Medicine http://www.naika.or.jp/imindex.html 